PROCEEDINGS OF THE

AMERICAN MATHEMATICAL SOCIETY

Volume 126, Number 8, August 1998, Pages 2493-2503

S 0002-9939(98)04762-5

\title{
THE GEOGRAPHY OF IRREDUCIBLE 4-MANIFOLDS
}

\author{
JONGIL PARK \\ (Communicated by Leslie Saper)
}

\begin{abstract}
In this paper we investigate the existence and the uniqueness problems for simply connected irreducible 4-manifolds. By taking fiber sums along an embedded surface of square 0 and by a rational blow-down procedure, we construct many new irreducible 4-manifolds which have infinitely many distinct smooth structures. Furthermore, we prove that all but at most finitely many lattice points lying in the non-positive signature region with $2 e+3$ sign $\geq 0$ are covered by these irreducible 4 -manifolds.
\end{abstract}

\section{INTRODUCTION}

As gauge theory (Donaldson theory and Seiberg-Witten theory) is developed, many problems and conjectures related to smooth 4-manifolds are solved, and it gives us a new insight into smooth 4-manifold theory.

In 1993, R. Fintushel and R. Stern introduced a surgical procedure, called rational blow-down, to compute the Donaldson series for simply connected regular elliptic surfaces with multiple fibers of relatively prime orders. The 'rational blowdown' means that if a smooth 4-manifold $X$ contains a certain configuration $C_{p}$ of transversely intersecting 2 -spheres whose boundary is the lens space $L\left(p^{2}, 1-p\right)$, then one can construct a new smooth 4 -manifold $X_{p}$ from $X$ by replacing $C_{p}$ with a rational ball $B_{p}$. They initially computed the Donaldson series of $X_{p}$ from the Donaldson series of $X$, and later they computed the Seiberg-Witten invariants of $X_{p}([\mathrm{FS} 2])$. A generalized version of their results is also obtained in the author's dissertation.

In this paper we apply these results to the geography problems for simply connected smooth irreducible 4-manifolds-namely, the existence problem and the uniqueness problem. We give partial answers for these problems. That is, we find many new examples of such 4-manifolds whose $\chi=\frac{b^{+}+1}{2}$ and $c_{1}^{2}$ lie in the wedge between $c_{1}^{2}=0$ and $c_{1}^{2}=2 \chi-6$ in the $\left(\chi, c_{1}^{2}\right)$-plane. Actually we construct new examples which cover all lattice points in this region. (The examples lying in the wedge were first found by R. Fintushel and R. Stern, and later by R. Gompf and A. Stipsicz.) Note that any such 4-manifold in this region cannot admit a complex structure with either orientation. We also investigate the uniqueness problem, i.e. the problem of finding infinitely many diffeomorphism types for a given pair $\left(\chi, c_{1}^{2}\right)$ lying in between $c_{1}^{2}=0$ and $c_{1}^{2} \leq 9 \chi$. By using the rational blow-down

Received by the editors January 23, 1997.

1991 Mathematics Subject Classification. Primary 57N13, 57R55.

Key words and phrases. Fiber sum, irreducible manifold, rational blow-down.

(C)1998 American Mathematical Society 
technique, we can compute Seiberg-Witten invariants of $X(p)$, where $X(p)$ is the result of $p$-surgery in the cusp neighborhood of a cusp fiber in $X$. Under a mild condition on $X$ making $X(p)$ simply connected, such $X(p)$ is not diffeomorphic, but is homeomorphic to $X$. In fact, for infinitely many $\left(\chi, c_{1}^{2}\right)$, we can find irreducible 4-manifolds containing a cusp fiber satisfying the mild condition, for example, by taking fiber sums. The main result we prove in this paper is

Theorem 1.1. All but at most finitely many lattice points satisfying $0 \leq c_{1}^{2} \leq 8 \chi$ in the $\left(\chi, c_{1}^{2}\right)$-plane can be realized as $\left(\chi, c_{1}^{2}\right)$ of a simply connected symplectic 4 manifold which has infinitely many distinct irreducible smooth structures.

\section{The GEOGRAPHY OF IRREDUCIBLE 4-MANIFOLDS}

The geography problems we are interested in studying are twofold, that is, which lattice points in the $\left(\frac{b^{+}+1}{2}, 3 \sigma+2 e\right)$-plane are 'populated' by simply connected smooth irreducible 4-manifolds (the existence problem) and if so, are there infinitely many distinct smooth 4-manifolds which are mutually homeomorphic (the uniqueness problem)? (We denote the signature of $X$ by $\sigma(X)$, and the Euler characteristic of $X$ by $e(X)$.) These coordinates are chosen because of their relation to complex surfaces where the holomorphic Euler characteristic $\chi$ and the Chern number $c_{1}^{2}$ satisfy $\chi=\frac{1}{12}\left(c_{1}^{2}+c_{2}\right)=\frac{b^{+}-b_{1}+1}{2}$ and $c_{1}^{2}=3 \sigma+2 e$. The geography problem for surfaces of general type has been studied extensively by algebraic surface theorists (see the Remarks below), and for topologists, the problems are to find constructions of new 4-manifolds and to be able to compute invariants (such as Donaldson invariants and Seiberg-Witten invariants) which can show that the result is an irreducible 4-manifold. Note that a smooth 4-manifold $X$ is called $i r$ reducible if $X$ is not a connected sum of other manifolds except for a homotopy sphere, i.e. if $X=X_{1} \sharp X_{2}$ implies that one of the $X_{i}$ is a homotopy sphere. One of the most powerful applications of gauge theory to 4-dimensional topology related to geography problems is that both the Donaldson invariants, denoted by $D_{X}$, and the Seiberg-Witten invariants, denoted by $S W_{X}$, for a connected sum manifold $X=X_{1} \sharp X_{2}$ with $b^{+}\left(X_{i}\right)>0(i=1,2)$ are identically zero. Hence $S W_{X} \neq 0$ (or $D_{X} \neq 0$ ) implies that $X$ is irreducible unless $X$ is homeomorphic to a blow-up manifold. Here are a few remarks on Figure 1:

1. The simply connected minimal complex surfaces of general type live in the dotted region determined by the "Noether line", $c_{1}^{2}=2 \chi-6(5 \sigma+3 e+12 \geq 0)$, and the "Bogomolov-Miyaoka-Yau line", $c_{1}^{2}=9 \chi(3 \sigma \leq e)$. A surface of signature 0 lies on the line $c_{1}^{2}=8 \chi$, and any surface of negative signature lies in the region $c_{1}^{2}<8 \chi$. It is also known that any lattice point lying in the region $2 \chi-6<c_{1}^{2}<8 \chi$ can be realized as $\left(\chi, c_{1}^{2}\right)$ of a minimal surface which is a genus two Lefschetz fibration $([\mathrm{P}])$.

2. Moishezon and Teicher constructed infinitely many simply connected minimal surfaces of positive signature (equivalently, lying in between $c_{1}^{2}=8 \chi$ and $\left.c_{1}^{2}=9 \chi\right)$. Chen and Xiao also constructed other simply connected minimal surfaces of positive signature which are hyperelliptic fibrations $([\mathrm{C}])$.

3. Any irreducible 4-manifold in the wedge between the "elliptic line", $c_{1}^{2}=0$, and the "Noether line", $c_{1}^{2}=2 \chi-6$, cannot admit a complex structure with either orientation, because it would violate either the Noether inequality or the Bogomolov-Miyaoka-Yau inequality. The examples lying in this wedge were found by Fintushel and Stern ([FS2]), and by Gompf and Stipsicz ([S]) 


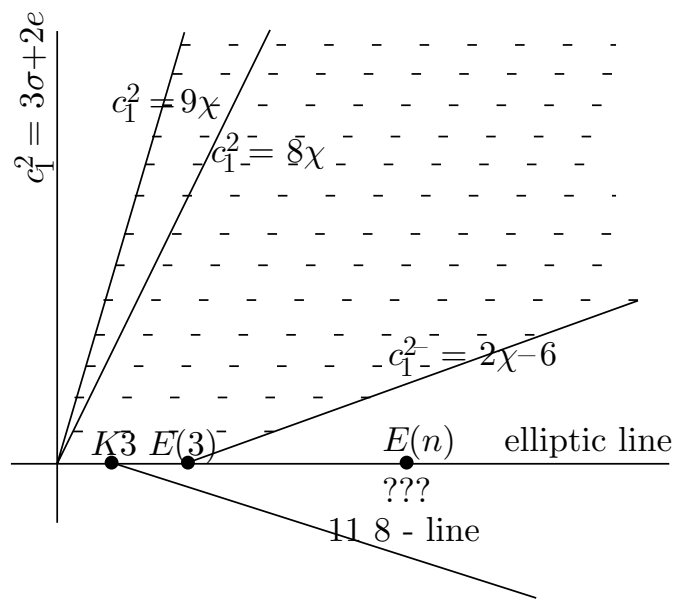

Figure 1

independently. We also construct new examples covering all lattice points in this wedge (see Theorem 2.7).

4. There are no known irreducible 4-manifolds lying in the elliptic line below $c_{1}^{2}<0$, and there is a conjecture that every smooth spin 4-manifold satisfies $\frac{b_{2}}{|\sigma|} \geq \frac{11}{8}$. Note that the rational blow-down procedure moves a manifold vertically upward, and the blowing up procedure moves a manifold vertically downward in Figure 1.

The main result (Theorem 2.8) in this paper will be obtained by using two surgical procedures, called a rational blow-down and a fiber sum, whose SeibergWitten invariants are computed.

Definition. Suppose a smooth 4 -manifold $X$ contains a configuration $C_{p}$ which is a smooth 4-manifold obtained by plumbing disk bundles over the 2 -sphere instructed by the linear diagram

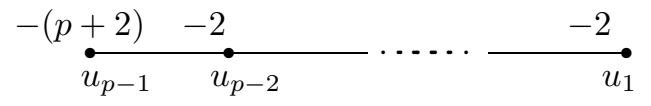

where each vertex $u_{i}$ represents a disk bundle over the 2-sphere with Euler class indicated by the label. The rational blow-down of $X$ is defined as a smooth 4manifold, denoted by $X_{p}$, obtained by replacing a configuration $C_{p}$ with a rational ball $B_{p}$. Note that this procedure is well defined, i.e. $X_{p}$ is uniquely constructed (up to diffeomorphism) from $X$ because each diffeomorphism of $\partial B_{p}=L\left(p^{2}, 1-p\right.$ ) extends over the rational ball $B_{p}$.

Fintushel and Stern ([FS2]) showed that there is a configuration $C_{p}$ in $E(n) \sharp(p-1) \overline{\mathbf{C P}}^{2}$ such that the rational blow-down along $C_{p}$ is diffeomorphic to $E(n ; p)$, the $p$-log transform on $E(n)$, and they computed the Donaldson and Seiberg-Witten invariants of simply connected elliptic surfaces with multiple fibers. Here $E(n)$ is the simply connected elliptic surface with no multiple fibers and holomorphic Euler characteristic $n$, and ' $p$-log transform on $E(n)$ ' is the result of removing a tubular neighborhood of a torus fiber in $E(n)$, say $T^{2} \times D^{2}$, and regluing 
it by a diffeomorphism

$$
\varphi: T^{2} \times \partial D^{2} \longrightarrow T^{2} \times \partial D^{2}
$$

such that the absolute value of the degree of the map

$$
\operatorname{proj}_{\partial D^{2}} \circ \varphi: p t \times \partial D^{2} \longrightarrow \partial D^{2}
$$

is $p$. Note that ' $p$-log transform on $E(n)$ ' is well defined, i.e. $E(n ; p)$ is uniquely determined up to diffeomorphism by the fact that if $\operatorname{proj}_{\partial D^{2}} \circ \varphi$ and $\operatorname{proj}_{\partial D^{2}} \circ \varphi^{\prime}$ have the same degree up to sign, then the resulting two manifolds are diffeomorphic.

Theorem 2.1 ([FS2]). The Seiberg-Witten invariants of $E(n ; p)$ are

$$
S W_{E(n ; p)}=S W_{E(n)} \cdot\left(e^{(p-1) f_{p}}+e^{(p-3) f_{p}}+\cdots+e^{-(p-1) f_{p}}\right),
$$

where $f_{p}$ is a multiple fiber obtained by $p$-log transform on $E(n)$.

In fact, one can extend the 'logarithmic transform' procedure to any 4-manifold which contains a cusp neighborhood. A cusp in a 4-manifold means a PL embedded 2 -sphere of self-intersection 0 with a single non-locally-flat point whose neighborhood is the cone on the right-hand trefoil knot, and we define a cusp neighborhood in a 4-manifold to be a manifold $N$ obtained by performing 0 -framed surgery on the trefoil knot in the boundary of the 4-ball. Note that since the trefoil knot is a fibered knot with a genus 1 fiber, $N$ is fibered by tori with one singular fiber which is a cusp. Hence one can perform ' $p$-log transform' on a regular torus fiber in $N$ exactly the same way as in $E(n)$, so that the theorem above is also true for any smooth 4-manifold containing a cusp neighborhood. Hence, by extending the notion of ' $p$-log transform' to any smooth 4-manifold containing a cusp neighborhood, Theorem 2.1 is easily extended as follows:

Corollary 2.1. Let $X(p)$ be the result of $p$-log transform in the neighborhood of a cusp, say $f$, in a smooth 4-manifold $X$. Then the Seiberg-Witten invariants of $X(p)$ are

$$
S W_{X(p)}=S W_{X} \cdot\left(e^{(p-1) f_{p}}+e^{(p-3) f_{p}}+\cdots+e^{-(p-1) f_{p}}\right),
$$

where $f_{p}$ is a multiple fiber in $X(p)$ obtained by $p$-log transform on $X$.

Proof. The proof is the same as the proof of Theorem 2.1 if each basic class $L$ of $X$ satisfies $f \cdot L=0$ ([FS2]). But, since genus $(f)=1$ and $f^{2}=0$, the condition $f \cdot L=0$ is automatically satisfied by the adjunction inequality

$$
f^{2}+|f \cdot L| \leq 2 \cdot \operatorname{genus}(f)-2
$$

Corollary 2.1 implies that a manifold $X$ and its $p$-log transform $X(p)$ have different smooth structures because they have different Seiberg-Witten invariants. This fact allows us to partially answer our uniqueness question above. Before going on, we quote a well-known theorem on $X(p)$, the $p$-log transform of $X$.

Theorem 2.2 ([FS1]). Let $X$ be a simply connected 4-manifold containing a cusp neighborhood $N$ whose complement $Z$ has $\pi_{1}(Z)=\mathbf{Z}_{q}, q \geq 1$, and $\pi_{1}(\partial N) \rightarrow \pi_{1}(Z)$ is surjective. Let $\Xi(X)$ be the class of 4-manifolds $\{X(p): p, q$ coprime, $p \neq 0\}$. Then:

(a) Each $X(p) \in \Xi(X)$ is simply connected.

(b) If $X$ is not spin or $q$ is even, all the manifolds in $\Xi(X)$ are homeomorphic.

(c) If $X$ is spin and $q$ is odd, then the manifolds $X(p)$ and $X\left(p^{\prime}\right)$ in $\Xi(X)$ are homeomorphic if and only if $p \equiv p^{\prime}(\bmod 2)$. 
Hence, by Theorem 2.2 and Corollary 2.1, any smooth 4-manifold containing a cusp neighborhood satisfying the hypothesis of Theorem 2.2 has infinitely many distinct smooth structures. For example, Fintushel and Stern showed by using Donaldson invariants that any algebraic surface containing a Brieskorn manifold $B(p, q, r)$ with $(p, q, r) \geq(2,3,7)$ has infinitely many diffeomorphism types ([FS1]). Note that if a smooth 4-manifold $X$ contains a cusp neighborhood $N$ with a cusp fiber $f$ and an embedded 2-sphere $S$ with $S \cdot S<0$ such that $S$ intersects the cusp fiber $f$ transversely in a single point, then $X$ satisfies the hypothesis of Theorem 2.2 $\left(\pi_{1}(Z)=1\right)$. In this case, we say that $X$ contains a nicely embedded cusp neighborhood. Now we construct more irreducible 4-manifolds which have infinitely many diffeomorphism types. First we define another topological surgery, called fiber sum.

Definition. Let $X$ and $Y$ be closed, oriented, smooth 4-manifolds containing a smoothly embedded surface $\Sigma$ of genus $g \geq 1$. Suppose $\Sigma$ represents a homology class of infinite order and of square zero, so that there exists a tubular neighborhood, say $D^{2} \times \Sigma$, in $X$ and $Y$. Let $X_{0}=X \backslash D^{2} \times \Sigma$ and $Y_{0}=Y \backslash D^{2} \times \Sigma$, and let $N=S^{1} \times \Sigma=\partial D^{2} \times \Sigma$ be the common boundary of $D^{2} \times \Sigma$. By choosing an orientation-reversing, fiber-preserving diffeomorphism

$$
\varphi: D^{2} \times \Sigma \longrightarrow D^{2} \times \Sigma
$$

and gluing $X_{0}$ to $Y_{0}$ along their boundary by the map $\varphi \mid: N \longrightarrow N$, we define a new oriented smooth 4-manifold $X \sharp_{\Sigma} Y$, called the fiber sum of $X$ and $Y$ along $\Sigma$. Note that there is an induced embedding of $\Sigma$ into $X \sharp_{\Sigma} Y$, well-defined up to isotopy, which represents a homology class of infinite order and of square zero.

Lemma 2.1. Suppose $X$ and $Y$ are simply connected smooth 4-manifolds containing a tubular neighborhood $D^{2} \times \Sigma$ of an embedded surface $\Sigma$. If $S^{1} \cong \partial D^{2} \times\{p t\}$ bounds a disk in $X_{0}=X \backslash D^{2} \times \Sigma$ or $Y_{0}=Y \backslash D^{2} \times \Sigma$, then $X \sharp_{\Sigma} Y$ is simply connected.

Proof. This fact follows from the Van Kampen's theorem:

$$
\pi_{1}\left(X \sharp_{\Sigma} Y\right)=\frac{\pi_{1}\left(X_{0}\right) * \pi_{1}\left(Y_{0}\right)}{N}
$$

where $*$ is a free product and $N$ is a normal subgroup generated by $\pi_{1}\left(\partial D^{2} \times \Sigma\right)$.

Lemma 2.2. Let $X$ and $Y$ be closed, oriented, smooth 4-manifolds containing an embedded surface $\Sigma$ of square zero. Then $c_{1}^{2}\left(X \sharp_{\Sigma} Y\right)=c_{1}^{2}(X)+c_{1}^{2}(Y)+8(g-1)$ and $\chi\left(X \sharp_{\Sigma} Y\right)=\chi(X)+\chi(Y)+(g-1)$, where $\chi=\frac{b^{+}-b_{1}+1}{2}$ and $g=\operatorname{genus}(\Sigma)$.

Proof. These follow from the facts that

$$
\begin{aligned}
e\left(X \sharp_{\Sigma} Y\right) & =e(X)-(2-2 g)+e(Y)-(2-2 g) \\
& =e(X)+e(Y)+4(g-1), \\
\sigma\left(X \sharp_{\Sigma} Y\right) & =\sigma(X)+\sigma(Y) .
\end{aligned}
$$

We quote a product formula for Seiberg-Witten invariants of a fiber sum manifold which provides an important tool for studying geography problems for irreducible 4-manifolds.

Theorem 2.3 ([MST]). Let $X$ and $Y$ be closed, oriented, smooth 4-manifolds containing a smoothly embedded surface $\Sigma$ of genus $g>1$. Suppose $\Sigma$ represents a homology class of infinite order and of square zero, and $b^{+}(X), b^{+}(Y) \geq 1$. If 
there are characteristic classes $l_{1} \in H^{2}(X ; \mathbf{Z})$ and $l_{2} \in H^{2}(Y ; \mathbf{Z})$ with $\left\langle l_{1},[\Sigma]\right\rangle=$ $\left\langle l_{2},[\Sigma]\right\rangle=2 g-2$ and with $S W_{X}\left(l_{1}\right) \neq 0$ and $S W_{Y}\left(l_{2}\right) \neq 0$, then there exists a characteristic class $k \in H^{2}\left(X \sharp_{\Sigma} Y ; \mathbf{Z}\right)$ with $\left.k\right|_{\partial D^{2} \times \Sigma}=\operatorname{proj}^{*}\left(k_{0}\right)$ for $k_{0} \in H^{2}(\Sigma ; \mathbf{Z})$ satisfying $\left\langle k_{0},[\Sigma]\right\rangle=2 g-2$, for which $S W_{X \sharp_{\Sigma} Y}(k) \neq 0$.

In case $\operatorname{genus}(\Sigma)=1$ (i.e. $\Sigma=$ torus), they also proved

Theorem 2.4 ([MMS]). Suppose $X$ and $Y$ contain a cusp neighborhood of a cusp fiber $f$. Then $S W$-basic classes of $X \sharp_{f} Y$ are given by

$$
\left\{K_{X}+K_{Y}+n \cdot f: K_{X}\left(K_{Y}\right) \text { is a basic class of } X(Y) \text { and } n=0, \pm 2\right\} \text {. }
$$

Remark. D. McDuff and T. Parker independently also showed a similar statement for symplectic manifolds by using Gromov invariants.

Theorem 2.5. The fiber sum of two minimal symplectic 4 -manifolds with $b^{+} \geq 2$ along a symplectic (or lagrangian) surface is also minimal symplectic.

Proof. It follows from Gompf's result and Lorek's result. That is, Gompf proved that a fiber sum along a symplectic (or lagrangian) surface is symplectic ([G]) and Lorek proved the minimality. Here is a sketch of Lorek's argument $([\mathrm{L}])$ : Suppose $X$ and $Y$ are minimal symplectic 4-manifolds containing a symplectic surface $\Sigma$ of square zero. For simplicity, we assume that a trivial disk bundle $D^{2} \times \Sigma$ over $\Sigma$ is contained in both $X$ and $Y$. For each point $t \in D^{2}$, remove a disk $D_{1}^{2} \subset D^{2}$ with center $(0,0)$ and $t \in \partial D_{1}^{2}$, and take a fiber sum $M_{t}:=X \sharp_{\Sigma} Y$ by gluing it along its boundary $\partial D_{1}^{2} \times \Sigma$. In this way we get a 6 -dimensional symplectic manifold $M$ which is a symplectic fibration over $D^{2}$ having one singular fiber $M_{(0,0)}=X \cup Y$ with $X \cap Y=\Sigma$ :

$$
M=\bigcup M_{t}= \begin{cases}X \sharp_{\Sigma} Y & t \neq(0,0) \in D^{2}, \\ X \cup Y & t=(0,0) \in D^{2} .\end{cases}
$$

Suppose $X \sharp_{\Sigma} Y$ is not minimal. Then there is a family $C_{t} \subset M_{t}$ of exceptional curves, which are assumed to be symplectically embedded because any embedded exceptional curve in a symplectic 4 -manifold with $b^{+} \geq 2$ can be symplectically embedded ([T]). Furthermore we may consider $C_{t}$ as a pseudo-holomorphic curve in $M_{t}$ by choosing a generic, $\omega_{t}$-tame, almost complex structure on $\left(M_{t}, \omega_{t}\right)$. Hence, by applying Gromov's compactness theorem for the family $C_{t}(t \rightarrow(0,0))$ of pseudoholomorphic curves in $M$, we get a limiting curve $C=C_{1} \cup \cdots \cup C_{k}$ lying in $M_{(0,0)}=$ $X \cup Y$ such that each $C_{i}$ is an embedded 2-sphere and $C^{2}=C_{1}^{2}+\cdots+C_{k}^{2}=-1$. We can assume that each $C_{i}(1 \leq i \leq k)$ lies in either $X$ or $Y$. Since there is no embedded sphere of positive square in a symplectic 4-manifold with $b^{+} \geq 2$, each $C_{i}$ satisfies $C_{i}^{2} \leq 0$. Thus there exists at least one $C_{i}$ in $X$ or $Y$ such that $C_{i}^{2}=-1$, which is a contradiction.

Theorem $2.6([\mathrm{~K}],[\mathrm{T}])$. If $X$ is a minimal symplectic 4 -manifold with $b_{2}^{+}(X) \geq 2$, then $X$ is irreducible.

These theorems enable us to partially solve our existence question.

Theorem 2.7. Every lattice point $\left(\chi, c_{1}^{2}\right)$ in the wedge between the elliptic line $\left(c_{1}^{2}=0\right)$ and the Noether line $\left(c_{1}^{2}=2 \chi-6\right)$ is realized by an infinite family of mutually homeomorphic, but distinct, simply connected smooth irreducible 4-manifolds. 
Proof. Since there is a nucleus $N^{\prime}$ in $B(2,3,11) \subset E(2)=K 3$ and $B(2,3,11) \subset$ $B(2,3,6 k-1)$ for $k \geq 2$, we consider a torus fiber sum $X(k, n) \equiv E(k) \sharp_{f} E(n-k)$ obtained by choosing a cusp $f$ in a cusp neighborhood $N \subset N^{\prime}$ in $B(2,3,6 k-1) \subset$ $E(k)$ and in $B(2,3,6 n-6 k-1) \subset E(n-k)$ :

$$
X(k, n)=N_{k} \cup_{\Sigma(2,3,6 k-1)} B(2,3,6 k-1) \sharp_{f} B(2,3,6 n-6 k-1) \cup_{\Sigma(2,3,6 n-6 k-1)} N_{n-k},
$$

where $N_{k}$ and $N_{n-k}$ are a neighborhood of a singular fiber and are sections in $E(k)$ and $E(n-k)$ respectively. Note that $X(k, n)$ and $E(n)$ have the same $\left(\chi, c_{1}^{2}\right)=$ $(n, 0)$, but they are not even homeomorphic if $n$ is even and $k$ is odd, because $E(n)$ is spin but $X(k, n)$ is not spin. Since an elliptic surface $E(k)$ can be obtained from a Horikawa surface $H(k)$ by rationally blowing up along two disjoint $C_{k}$ 's ([FS2]) and a Horikawa surface $H(k)$ contains a cusp neighborhood ([S]), it follows that $E(k) \backslash N$ contains two disjoint configurations $C_{k}$. Hence $X(k, n)$ also contains two disjoint configurations $C_{k}$. Furthermore, since the nucleus $N^{\prime}$ contains a 2-sphere of square -2 intersecting $f$ transversely at one point, by taking a fiber sum along $f, X(k, n)$ contains a 2-sphere of square -4 coming from those two 2-spheres which intersect $f$ at one point. By rationally blowing down these configurations, $C_{k}$ and $C_{4}$, we can fill every lattice point in the wedge. Explicitly, if $1 \leq c_{1}^{2} \leq n-5$, rationally blow down along a configuration $C_{k}$ for $4 \leq k \leq n-2$. If $n-4 \leq c_{1}^{2} \leq 2 n-9$ and $c_{1}^{2}$ is even (odd), rationally blow down twice along two $C_{k}$ 's (two $C_{k}$ 's and one $C_{4}$ ). Finally, if $c_{1}^{2}=2 n-8(2 n-7)$, rationally blow down four times (five times) along two $C_{n-2}$ 's and two $C_{4}$ 's (two $C_{n-2}$ 's and three $C_{4}$ 's). Irreducibility of these rational blow-down manifolds follows from Theorem 2.4 and the fact that such manifolds cannot be blow-up manifolds. (Otherwise, there would exist $S W$ basic classes $\overline{K_{1}}, \overline{K_{2}}$ satisfying $\left(\overline{K_{1}}-\overline{K_{2}}\right)^{2}=-4$ such that $\left.\overline{K_{i}}\right|_{X_{0}}(i=1,2)$ extends to a basic class $K_{i}(i=1,2)$ for $X(k, n)$. Since $\left(K_{1}-K_{2}\right)^{2}=0$ (Theorem 2.4) and $C_{k}$ is negative definite,

$$
\begin{aligned}
0 & =\left(\left.K_{1}\right|_{X_{0}}-\left.K_{2}\right|_{X_{0}}\right)^{2}+\left(\left.K_{1}\right|_{C_{k}}-\left.K_{2}\right|_{C_{k}}\right)^{2} \\
& =\left(\overline{K_{1}}-\overline{K_{2}}\right)^{2}+\left(\left.K_{1}\right|_{C_{k}}-\left.K_{2}\right|_{C_{k}}\right)^{2} \\
& \leq\left(\overline{K_{1}}-\overline{K_{2}}\right)^{2}=-4
\end{aligned}
$$

which is a contradiction.) Since all such rational blow-down manifolds still contain a nicely embedded cusp neighborhood, Corollary 2.1 and Theorem 2.2 imply that all these manifolds have infinitely many distinct smooth structures.

Here are a few remarks on this theorem:

1. Fintushel and Stern proved this theorem by using twisted fiber sums. Gompf ([G]) and Stipsicz ([S]) also showed that any lattice point with $c_{1}^{2}$ even in the wedge can be realized as $\left(\chi, c_{1}^{2}\right)$ of simply connected, irreducible, symplectic manifolds. Stipsicz' examples are $X \sharp_{f} E(n)$, where $X$ is a Horikawa surface with $c_{1}^{2}=2 \chi-6$.

2. In fact, we can show that every lattice point in the wedge is realized as $\left(\chi, c_{1}^{2}\right)$ of simply connected, irreducible, symplectic manifolds by using nine symplecti-breal cally embedded 2-spheres of square -4 in $E(4)$ and by a slight modification of Stipsicz' examples. That is, first consider a torus fiber sum manifold $Y \equiv X \sharp_{f^{\prime}} E(4)$, where $f^{\prime}$ is an embedded torus in the cusp neighborhood contained in a Milnor fiber $B(2,3,23) \subset E(4)$ and $X$ is a Horikawa surface as above. Since such an embedded torus $f^{\prime}$ is lagrangian ([S]), $Y$ is 
a symplectic manifold by Gompf's argument $([\mathrm{G}])$. Note that $Y$ still contains a symplectically embedded 2 -sphere of square -4 (a configuration $C_{4}$ ). Since the rational blow-down along $C_{4}$ is equivalent to a fiber sum with a quadric in $\mathbf{C P}^{2}$, the rational blow-down manifold $Y_{4}$ of $Y$ is again a symplectic irreducible 4-manifold. Now, as in Stipsicz' examples, construct torus fiber sum manifolds $Y_{4} \sharp_{f} E(n)$. Then any lattice point with $c_{1}^{2}$ odd in the wedge is realized by these symplectic manifolds. Irreducibility follows from the same argument as in the proof above, and simply-connectedness follows from Lemma 2.1 because $S^{1}=\partial D^{2}$ bounds a disk $S^{2} \backslash D^{2}$, where $S^{2}$ is a transversely intersecting 2 -sphere with a fiber $f$ at one point in $E(n)$. Furthermore, since they contain a nicely embedded cusp neighborhood lying in $E(n)$, all these symplectic manifolds have infinitely many distinct smooth structures.

3. Each of the manifolds constructed in the proof above has more than one $S W$-basic class. Actually one can construct infinitely many irreducible 4manifolds which have, up to sign, two (three, four, .. ) $S W$-basic classes by using slightly different examples.

Let $\Omega$ be the set of all lattice points in the wedge between $c_{1}^{2}=0$ and $c_{1}^{2} \leq 2 \chi-6$. Then we have

Corollary 2.2. If $X$ is a simply connected irreducible symplectic 4-manifold which contains a symplectic (or lagrangian) torus in a cusp neighborhood, then each lattice point in $\left(\chi(X), c_{1}^{2}(X)\right)+\Omega$ is realized by a simply connected irreducible symplectic 4-manifold which has infinitely many diffeomorphism types.

Proof. By taking a torus fiber sum of $X$ with symplectic manifolds constructed in Remark 2 above, we get a family of desired irreducible symplectic manifolds. Irreducibility follows from Theorem 2.5 and Theorem 2.6. Simply-connectedness follows from Lemma 2.1 because symplectic manifolds constructed in Remark 2 above contain a 2 -sphere transversely intersecting a fiber $f$ at one point in $E(n)$.

Corollary 2.3. Suppose $X$ is a simply connected irreducible 4-manifold containing a cusp neighborhood. If $\left(K_{X}-K_{X}^{\prime}\right)^{2} \geq 0$ for all $S W$-basic classes $K_{X}, K_{X}^{\prime}$ of $X$, then each lattice point in $\left(\chi(X), c_{1}^{2}(X)\right)+\Omega$ is also realized by a simply connected irreducible 4-manifold which has infinitely many diffeomorphism types.

Remark. The hypothesis $\left(K_{X}-K_{X}^{\prime}\right)^{2} \geq 0$ in Corollary 2.3 guarantees the irreducibility of a fiber sum manifold, but it seems likely to be unnecessary.

Now consider a Milnor fiber $B(p, q, p q-1)=\left\{\left(z_{1}, z_{2}, z_{3}\right) \in \mathbf{C}^{3}: z_{1}^{p}+z_{2}^{q}+z_{3}^{p q-1}=\right.$ $\epsilon$, for $\epsilon>0\}$, which has a natural compactification (by adding a complex curve at infinity) as a complete intersection in a weighted homogeneous space. Note that the singularities of this compactification can be resolved to obtain a simply connected algebraic surface $X(p, q, p q-1)$.

Example. The Milnor fiber $B(2,2 n+1,4 n+1)$ is contained in the simply connected algebraic surface $X(2,2 n+1,4 n+1)$, which is diffeomorphic to

$$
B(2,2 n+1,4 n+1) \cup_{\Sigma(2,2 n+1,4 n+1)} T(2,2 n+1) .
$$

$T(2,2 n+1)$ is the manifold obtained from +1 surgery on the $(2,2 n+1)$ torus knot, and it contains an obvious surface $T$ of genus $n$ and square +1 . The canonical class 
$K_{X}$ of $X(2,2 n+1,4 n+1)$ is represented by a multiple of $T$. Let

$$
X^{\prime}=X(2,2 n+1,4 n+1) \sharp \overline{\mathbf{C P}}^{2}
$$

be the manifold obtained by blowing up at a point in $T$, so that

$$
X^{\prime} \cong B(2,2 n+1,4 n+1) \cup_{\Sigma(2,2 n+1,4 n+1)} C(2,2 n+1),
$$

where $C(2,2 n+1)$ is the blow up of $T(2,2 n+1)$. In $X^{\prime}$ there is an embedded surface $\Sigma$ representing $T-e$, and $\Sigma$ has genus $n$ and self-intersection 0 . Since $\Sigma$ is symplectically embedded, by taking a fiber sum of $X^{\prime}$ with itself along $\Sigma$ (Figure 2), we get a simply connected symplectic 4-manifold $Z \equiv X^{\prime} \sharp_{\Sigma} X^{\prime}$ which satisfies the hypothesis of Theorem 2.3. Furthermore, $Z$ is irreducible if $n$ is odd. Note that the irreducibility of $Z$ follows from Theorem 2.3 and the fact that $Z$ is spin because each part of the following Figure 2 is spin. (The middle part, $C(2,2 n+1) \#_{\Sigma}$ $C(2,2 n+1)$, is embedded in the elliptic surface $E(n+1)$. Note that $E(n+1)$, as a genus $n$ Lefschetz fibration, can be constructed as follows:

$$
E(n+1) \cong Q \cup_{\Sigma(2,2 n+1,4 n+1)} C(2,2 n+1) \sharp_{\Sigma} C(2,2 n+1) \cup_{\Sigma(2,2 n+1,4 n+1)} Q
$$

where $Q$ is the canonical resolution of singularity of $z_{1}^{2}+z_{2}^{2 n+1}+z_{3}^{4 n+1}=0$ in $\mathbf{C}^{3}$.)

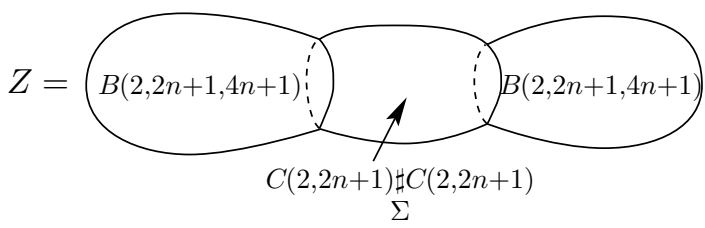

Figure 2

Finally, by using a simply connected, irreducible, symplectic manifold $Z$ constructed as above, we prove the main result of this paper:

Theorem 2.8. There is a linear function $y=f(x)$ such that any lattice point $(a, b)$ satisfying $b \leq f(a)$ in the first quadrant can be realized as $\left(\chi, c_{1}^{2}\right)$ of a simply connected symplectic 4-manifold which has infinitely many distinct irreducible smooth structures. In particular, all lattice points $(a, b)$ except at most finitely many in the region $0 \leq c_{1}^{2} \leq 8 \chi$ (i.e. $2 e+3 \operatorname{sign} \geq 0$ and sign $\left.\leq 0\right)$ satisfy $b \leq f(a)$.

Proof. Choose a simply connected minimal surface, say $Y$, of positive signature which is a hyperelliptic fibration with odd genus over $\mathbf{P}^{1} \cong S^{2}$. (One may choose a minimal surface constructed by Xiao and Chen $([\mathrm{C}])$ for $Y$.) Let $\Sigma$ be a fiber of $Y$ and let $g=\operatorname{genus}(\Sigma)$. Take any irreducible symplectic 4-manifold $Z$ which contains a symplectically embedded surface $\Sigma$ satisfying $\Sigma^{2}=0$ and also contains a symplectic (or lagrangian) torus $f$ in a cusp neighborhood $N$ satisfying $N \cap \Sigma=\emptyset$. (Such an irreducible symplectic 4-manifold exists! See the example constructed above.) Note that since

$$
c_{1}^{2}\left(Y \sharp_{\Sigma} Z\right)-8 \chi\left(Y \sharp_{\Sigma} Z\right)=\left[c_{1}^{2}(Y)-8 \chi(Y)\right]+\left[c_{1}^{2}(Z)-8 \chi(Z)\right]
$$

and

$$
\left[c_{1}^{2}(Y)-8 \chi(Y)\right]>0
$$


there exists an integer $k>0$ such that $X \equiv \overbrace{Y \sharp_{\Sigma} \cdots \sharp_{\Sigma} Y}^{k} \sharp_{\Sigma} Z$ has a positive signature. Since a manifold $Y$ contains a 2 -sphere $S^{2}$ intersecting transversely a fiber $\Sigma$ at one point, Lemma 2.1 implies that the fiber sum manifold $X$ is simply connected. Let $\Omega$ be the set of all lattice points in the wedge between $c_{1}^{2}=0$ and $c_{1}^{2} \leq 2 \chi-6$. Then Corollary 2.2 implies that each lattice point in $\left(\chi(X), c_{1}^{2}(X)\right)+\Omega$ is represented by a simply connected irreducible symplectic 4-manifold which has infinitely many diffeomorphism types. The same is true for $X \sharp_{f} X, X \sharp_{f} X \sharp_{f} X, \ldots$ So define $y=f(x)$ by

$$
f(x)=c_{1}^{2}(X) / \chi(X) \cdot\left[x-c_{1}^{2}(X) / 2-\chi(X)-3\right]+2 c_{1}^{2}(X)
$$

Then each lattice point $(a, b)$ satisfying $b \leq f(a)$ in the first quadrant is realized as $\left(\chi, c_{1}^{2}\right)$ of a simply connected irreducible symplectic 4 -manifold $\overbrace{X \sharp_{f} \cdots \sharp_{f} X}^{n} \sharp_{f} W$, for some $n \in \mathbf{Z}$ and a manifold $W$ constructed in Remark 2 after the proof of Theorem 2.7. Note that the irreducibility of such manifolds follows from Theorem 2.5 and Theorem 2.6 again. The second statement follows from the fact that the slope of $f(x), c_{1}^{2}(X) / \chi(X)$, is greater than 8 .

Remarks. 1. Note that there are still many lattice points in the region $y>f(x)$ which are realized as $\left(\chi, c_{1}^{2}\right)$ of $\overbrace{X \sharp_{f} X \sharp_{f} \cdots \sharp_{f} X}^{n} \sharp_{f} W$, for some $W \in \Omega$ and $n \in \mathbf{Z}$. Furthermore, we do not claim that the $f(x)$ constructed in the proof above is the best choice. In fact, one may choose better $y=f(x)$ having the same property by choosing another surface $X$ in the proof.

2. All such manifolds constructed in the proof above are likely to be non-spin because Chen's minimal surface $Y$ and a rational ball $B_{4}$ are non-spin.

We close this paper by suggesting the following problems:

Problem 1. Is every lattice point in the non-positive signature region realized as $\left(\chi, c_{1}^{2}\right)$ of an irreducible 4-manifold which has infinitely many diffeomorphism types? More generally, is it true for each lattice point in between the elliptic line $\left(c_{1}^{2}=0\right)$ and the Bogomolov-Miyaoka-Yau line $\left(c_{1}^{2}=9 \chi\right)$ ?

Problem 2. Which lattice points in the non-positive signature region are realized by irreducible spin 4-manifolds?

\section{ACKNOWLEDGEMENT}

The author would like to thank Professor Ronald Fintushel for guidance and help while working on this problem. He would also like to thank a referee for pointing out errors in the previous version of this paper.

\section{REFERENCES}

[C] Z. Chen, On the geography of surfaces: Simply connected minimal surfaces with positive index, Math. Ann. 277, 141-164 (1987). MR 88c:14057

[FS1] R. Fintushel and R. Stern, Surgery in cusp neighborhoods and the geography of irreducible 4-manifolds, Inventiones Math. 117, 455-523 (1994). MR 95f:57040

[FS2] R. Fintushel and R. Stern, Rational blowdowns of smooth 4-manifolds, J. Diff. Geo., to appear.

[G] R. Gompf, A new construction of symplectic manifolds, Annals of Math. 142, 527-595 (1995). MR 96j:57025 
[K] D. Kotschick, The Seiberg-Witten invariants of symplectic four-manifolds [after C.H. Taubes], Seminaire Bourbaki. 48eme annee, no 812 (1995-96), Astérisque, no. 241, Soc. Math. France, Paris, 1997, pp. 195-220. CMP 98:01

[L] W. Lorek, private communication.

[MMS] J. Morgan, T. Mrowka and Z. Szabó, Product formulas along $T^{3}$ for Seiberg-Witten invariants, in preparation.

[MST] J. Morgan, Z. Szabó and C. Taubes, A product formula for the Seiberg-Witten invariants and the generalized Thom conjecture, J. Diff. Geo. 44, 706-788 (1996). MR 97m:57052

[P] U. Persson, An introduction to the geography of surfaces of general type, Proc. Symp. Pure Math. vol 46, 195-217 (1987). MR 89a:14057

[S] A. Stipsicz, A note on the geography of symplectic manifolds, Turkish J. Math. 20 no $\mathbf{1}$, 135-139 (1996). MR 97m:57035

[T] C. Taubes, The Seiberg-Witten and the Gromov invariants, Mathematical Research Letters 2, 221-238 (1995). MR 96a:57076

Department of Mathematics, University of California Irvine, Irvine, California 926973875

E-mail address: jpark@math.uci.edu

Current address: Department of Mathematics, Kon-Kuk University, Kwangjin-gu Mojin-dong 93-1, Seoul 143-701, Korea

E-mail address: jipark@kkucc.konkuk.ac.kr 